\title{
Contracepção Hormonal segundo a Ótica do Estudante de Medicina: Mais um Desafio para o Ensino Médico Brasileiro?
}

\author{
Hormonal Contraception from the \\ Perspective of Medical Students: Another \\ Challenge for Brazilian Medical Education?
}

Margareth Rocha Peixoto Giglio ${ }^{I}$ Larissa Cruvinel Andrade ${ }^{I}$ Giovana Mahamed Daher ${ }^{I}$ Marilia Oliveira Ribeiro ${ }^{I}$ Marco Aurélio Albernaz

\section{PALAVRAS-CHAVE}

- Anticoncepcional;

- Transtonos de Enxaqueca;

- Tromboembolia;

- Hipertensão;

- Hábito de Fumar;

- Educação Médica.

\section{KEYWORDS}

- Contraceptives Agents;

- Migraine Disorders;

- Thromboembolism;

- Hypertension;

- Smoking;

- Medical Education.

Recebido em: 03/12/2014

Aprovado em: 18/04/2015

\begin{abstract}
RESUMO
Introdução: Desde a década de 1960, vêm-se utilizando anticoncepcionais orais como forma efetiva de contracepção. De modo a uniformizar e guiar a prescrição de tais medicamentos em situações especiais, a Organização Mundial da Saúde (OMS) lançou o guia Critérios de elegibilidade para o uso de contraceptivos. Objetivo: Este estudo objetiva avaliar os conhecimentos de internos de Medicina de uma universidade a respeito da prescrição de anticoncepcionais hormonais de acordo com o Guia da OMS. Métodos: Foi realizado um estudo transversal, descritivo, com alunos do quinto e sexto anos de Medicina de uma universidade em Goiânia (GO) por meio da aplicação de um questionário com perguntas técnicas sobre indicações e contraindicações de determinados métodos contraceptivos em situações especiais. Foi realizada análise univariada, e os dados foram dispostos em tabelas de frequência. Resultados: Responderam ao questionário $92 \%$ dos acadêmicos, dos quais $72 \%$ afirmaram se sentir aptos a prescrever anticoncepcionais hormonais (ACH). Os acertos variaram de 3\%, na prescrição correta em hipertensas leves, a 27\%, em mulheres com cefaleia leve sem sinais focais. Conclusão: A maioria dos alunos se sentia apta a prescrever contraceptivos hormonais, mas grande parte deles apresenta conhecimento inconsistente quando colocada em situações especiais presentes na prática clínica. Considerando-se que o interno dentro em breve ingressará no mercado de trabalho, propõe-se uma revisão do plano de ensino de forma a dar mais ênfase ao tema em questão.
\end{abstract}

\begin{abstract}
Introduction: Since the 1960s oral contraceptives have been used as an effective form of contraception. In order to standardize and guide the prescription of these drugs in special situations, the WHO launched the guide "WHO Eligibility Criteria for use of hormonal contraceptives". Objective: This article aims to evaluate $5^{\text {th }}$ and $6^{\text {th }}$ year students' knowledge about the prescription of hormonal contraceptives according to the WHO guidelines. Methods: A cross-sectional survey was administered to $5^{\text {th }}$ and $6^{\text {th }}$ year medicine students from a University in Goiânia (GO). Descriptive statistics were used to illustrate the correct prescription of hormonal contraceptive methods in special situations. Univariate analysis was performed and the data were arranged in frequency tables. Results: Of the 92\% of the students who answered the questionnaire, $72 \%$ felt able to prescribe hormonal contraceptive methods. Correct answers ranged from 3\% in the correct prescription to mild hypertensive patients, to $27 \%$ to women with a mild headache and without focal signs. Conclusion: Most of the student respondents felt able to prescribe hormonal contraceptives. However many of them had inconsistent knowledge when facing special situations of clinical practice. Given the importance of the issue at hand and considering that these students will soon be working as doctors, we suggest a review of the undergraduate medical curriculum.
\end{abstract}




\section{INTRODUÇÃO}

O uso de contraceptivos hormonais mudou o paradigma da sexualidade feminina, sendo o aspecto mais importante da revolução sexual verificada na década de 1960 ao permitir a dissociação entre sexo e gravidez. Entretanto, embora esse recurso esteja presente na vida moderna há mais de 50 anos, a medicina baseada em evidências, aliada à prática clínica, comprova que muitas mulheres ainda receiam o uso de métodos anticoncepcionais hormonais $(\mathrm{ACH})$ por desconhecimento e preconceitos ${ }^{1}$. Pelos mesmos motivos, profissionais de saúde deixam de prescrever ou orientar a contracepção hormonal ${ }^{2,3,4}$.

Há de se entender o papel dos hormônios isoladamente na contracepção, para compreender a evolução que os contraceptivos hormonais tiveram desde que foram lançados. $\mathrm{O}$ efeito anticoncepcional dos ACH vem da ação dos progestágenos, similares em quase todas as pílulas utilizadas nos $\mathrm{ACH}$. Os estrogênios, na atualidade o etinilestradiol (EE) e o valerato de estradiol, estão nos contraceptivos combinados para manter o endométrio estabilizado, evitar sangramentos irregulares e contrapor os efeitos androgênicos dos progestagênios ${ }^{5,6}$.

Contudo, são os compostos estrogênicos os principais responsáveis pelos efeitos colaterais dos $\mathrm{ACH}$, tais como o tromboembolismo, a piora da migrânea e o sinergismo com o tabagismo e a hipertensão arterial na doença cardiovascular. Quanto maior a dose dos estrógenos do $\mathrm{ACH}$, maiores são esses efeitos.

Assim, na evolução dos $\mathrm{ACH}$, procurou-se diminuir cada vez mais a dose do estrogênio, até obter preparados só com progestagênios. Buscou-se, ainda, encontrar progestágenos com efeitos menos androgênicos e mais toleráveis, viabilizar formas de administração alternativas à oral e em regimes modificados e, mais recentemente, formulações com efeitos extraconceptivos ${ }^{6}$.

Diante de tamanha evolução farmacológica, depara-se com uma ampla variedade de formulações no mercado. Sendo assim, amplia-se o rol de opções para o médico e a paciente, em conjunto, definirem a melhor opção terapêutica no que tange às formas de administração, esquemas posológicos, princípios ativos e dosagens. Torna-se possível, portanto, individualizar cada caso, adequando o medicamento à mulher, $\mathrm{e}$ não mais a mulher ao medicamento ${ }^{7,3}$.

Entretanto, o conhecimento dos riscos associados ao uso dos anticoncepcionais hormonais deve ser pedra angular do médico assistente e/ou do ginecologista que prescrevem. Entre eles se destaca o aumento do risco de eventos tromboembólicos e doenças cardiovasculares em tabagistas, devendo-se fazer uso parcimonioso em mulheres hipertensas, diabéticas e/ou com quadros migranosos ${ }^{7,1,8}$.

Para facilitar a escolha do melhor anticoncepcional para cada mulher de forma individualizada, a Organização Mundial da Saúde (OMS) lançou, em 1996, um guia de orientação denominado Critérios de elegibilidade para o uso de contraceptivos ${ }^{9}$. Contudo, há evidências de que nem todos os médicos e estudantes de Medicina têm amplo conhecimento do manual. Além disso, a literatura tem mostrado que médicos e estudantes, em especial os internos (quinto e sexto anos de graduação), muitas vezes, se sentem inseguros na abordagem e orientação do uso e escolha do método mais adequado a determinada mulher?. Apesar de não haver estudos que evidenciem uma correlação direta entre o conhecimento do profissional e a aderência da paciente à contracepção hormonal ${ }^{1}$, é razoável presumir que o médico e os serviços de saúde têm grande impacto na decisão de aderir ou não ao uso de algum método ${ }^{2,3,4}$.

Um estudo realizado em Pittsburgh com estudantes de Medicina mostrou que essa insegurança é consequência de dificuldades decorrentes do déficit de conhecimento a respeito desse assunto, atribuído principalmente à pequena carga horária destinada ao tópico "contracepção" e à falta de prática na prescrição durante o curso médico ${ }^{4}$. Existem evidências de que a falta de conhecimentos a respeito da contracepção hormonal é maior nas escolas dos países em desenvolvimento e subdesenvolvidos - justamente nos locais onde é necessário maior controle da natalidade ${ }^{4}$.

Este trabalho teve como objetivo principal avaliar o conhecimento dos alunos do internato de Medicina de uma universidade de Goiás sobre a contracepção hormonal nas situações especiais de migrânea, tabagismo, tromboembolismo e hipertensão arterial, de acordo com os critérios de elegibilidade da OMS.

\section{MÉTODO}

Foi realizado um estudo transversal, descritivo, que incluiu todos os alunos que cursavam o internato em 2014/2 que quiseram participar e assinaram o Termo de Consentimento Livre e Esclarecido. O estudo contou com um total de 165 alunos - cerca de $92 \%$ dos 179 alunos matriculados no internato (do nono ao $12^{\mathrm{o}}$ período).

O índice de perdas foi de 7,8\% em virtude da ausência do aluno durante a aplicação dos questionários ou recusa em participar do estudo. Nenhum questionário foi excluído após a coleta de dados.

A coleta dos dados se deu entre os meses de julho e agosto de 2014, com aplicação do questionário por pesquisadoras treinadas após orientação acerca do objetivo do estudo e das 
perguntas do questionário. Cada aluno respondeu ao questionário sem pesquisas a livros, documentos ou internet.

O questionário constava de perguntas abertas e fechadas, de cunho epidemiológico e referente à segurança/insegurança na contracepção e motivos atribuídos a isso. Alm disso, Havia também oito perguntas técnicas fechadas sobre contracepção nas situações de risco: migrânea, HAS, tabagismo e tromboembolismo. Essas perguntas foram baseadas nos critérios de elegibilidade dos contraceptivos da OMS.

Cada questão sobre situações técnicas relativas à prescrição dos ACH era de múltipla escolha e contava com o mesmo grupo de opções a serem assinaladas: (1) anticoncepcional oral combinado; (2) anticoncepcional com progesterona isolada; (3) DIU de progesterona; (4) injetável mensal; (5) injetável trimestral;(6) anticoncepção hormonal combinada transdérmica; (7) anticoncepção hormonal combinada via vaginal; (8) nenhuma das respostas anteriores. $\mathrm{O}$ aluno deveria, nessa segunda parte, ser capaz de fazer a leitura do caso e prescrever corretamente todos os métodos permitidos à situação, ou fazê-lo de forma parcial, identificando as contraindicações relativas e absolutas para cada situação.

Os dados foram digitados em banco de dados confeccionado no software SPSS 19.0 e analisados por meio de análise univariada, apresentada em tabelas de frequência.

O presente estudo foi apreciado e aprovado pelo Comitê de Ética e Pesquisa da PUC-GO, não havendo qualquer conflito de interesses a ser declarado.

\section{RESULTADOS}

O estudo analisou 165 internos, com média de idade de 24,4 anos, mediana de 24 anos e idade mínima e máxima de 20 anos e 41 anos, respectivamente. Observa-se que $77 \%$ já haviam passado pelo estágio em Ginecologia no internato, com todos os alunos já tendo cursado a disciplina de Ginecologia e Obstetrícia no quarto ano.

Setenta e seis por cento dos estudantes avaliados usavam algum método contraceptivo, seja ele hormonal ou de barreira, e, $81 \%$ dos internos consideraram que tiveram informação adequada sobre concepção durante o internato, com cerca de $70 \%$ deles se sentindo aptos a prescrever ACHs. Oitenta e quatro por cento se recordam das discussões sobre os critérios de elegibilidade para prescrição do ACH da OMS.

Quando confrontados com perguntas que se referiam ao melhor método a utilizar em situações especiais, houve um índice de erro de 90,3\% quando da indicação do método contraceptivo correto para pacientes hipertensas graves. Para hipertensas leves, o índice de acerto, levando-se em conta os critérios de elegibilidade, foi de 3\%, com 97\% dos alunos indicando métodos combinados de estrogênio e progestagênios ou contraindicando de forma absoluta o uso de qualquer método contraceptivo.

No caso de pacientes com antecedentes pessoais e familiares de trombose venosa, os índices de acerto foram ambos de $12 \%$. Já no caso de antecedentes pessoais de trombose arterial ou tromboembolismo pulmonar, os acertos foram de $20 \%$.

As maiores taxas de acertos ocorreram quando os estudantes foram confrontados com a situação de uma paciente no menacme com cefaleia moderada e sem sinais focais, correspondendo a $27 \%$ das respostas corretas. No caso de paciente com sinais de migrânea com aura (sinais focais) e pacientes tabagistas, maiores de 35 anos, os acertos correspondem, respectivamente, a $13 \%$ e $11 \%$.

\section{DISCUSSÃO}

A contracepção hormonal é o método mais usado em todo o mundo como forma de planejamento familiar, particularmente entre as brasileiras. Entretanto, os riscos e benefícios inerentes ao método não são de conhecimento pleno entre profissionais e graduandos da área da saúde $\mathrm{e}^{7,4,10,11,12}$.

O presente estudo procurou avaliar o conhecimento dos alunos sobre a prescrição de contraceptivos hormonais em situações especiais de saúde, bem como o correto reconhecimento de suas respectivas categorias segundo os critérios elaborados pela OMS. De acordo com os critérios de elegibilidade da OMS, condições de HAS grave e/ou leve, tromboembolismo pulmonar e trombose arterial, cefaleia com aura e tabagismo em maiores de 35 anos são consideradas como categorias 3/4 (riscos superam os beneficios/método que não deve ser usado) para formulações com estrogênio. Já antecedente pessoal ou familiar de primeiro grau de tromboflebite e cefaleia sem aura são categorizados como 1/2 (sem restrição ao uso/benefícios superam os riscos) para preparados combinados.

A análise das respostas mostrou que os acadêmicos tiveram grande dificuldade em diferenciar os casos classificados como categorias $1 / 2$ dos de categoria $3 / 4$. Tal fato levou a um maior índice de erro nos casos considerados de menor gravidade, já que nas respostas muitos deixaram de indicar as formulações que contêm estrogênio quando poderiam fazê-lo, o que reduziu o arsenal de possibilidades para as pacientes.

O grande número de erros encontrado nas respostas fechadas, ao se considerar correta a questão apenas quando todas as opções certas foram marcadas, mostra que os alunos do internato da universidade estudada não estão aptos a prescrever contraceptivos hormonais nas situações especiais avaliadas, apesar de $72 \%$ deles referirem se sentir aptos a fazer essa prescrição. Por outro lado, o maior acerto das respostas, quando consideradas individualmente, mostra conhecimento fragmentado e sem segurança sobre o assunto entre os alu- 
nos pesquisados, apesar de $74 \%$ dos internos referirem que já tinham tido discussão sobre os critérios da OMS e todos os alunos já terem cursado a disciplina de Ginecologia no quarto ano do curso médico.

As principais causas sinalizadas pelos acadêmicos para as debilidades encontradas foram: falta de carga horária para o assunto (50\%), falta de qualidade das aulas (21\%) e falta de tempo para discussão (14\%).

Outro aspecto observado nas respostas é que os acadêmicos não conseguem diferenciar plenamente os efeitos dos estrogênios e progestágenos nos preparados contraceptivos, deixando de prescrever um preparado só de progestagênio em situações em que estava contraindicado apenas o estrogênio. Da mesma forma, a deficiência se deu em relação à via de utilização $0^{2,3}$.

Como exemplo, em relação a trombose arterial e embolia pulmonar, o estudo mostrou que $98 \%$ dos alunos não prescreveriam $\mathrm{ACH}$ oral. Contudo, $4 \%$ e $8 \%$ prescreveriam as vias transdérmica e vaginal, respectivamente, esquecendo que essas vias contêm estrogênios. Já 85\%, 59\% e 43\% não prescreveriam, respectivamente, o injetável trimestral, o progestágeno isolado oral e o Mirena - preparados que podem ser usados nessas situações com segurança, pois não afetam significativamente o sistema de coagulação.

A influência dos esteroides sexuais no sistema cardiovascular já é bem estabelecida, haja vista que os vasos sanguíneos possuem receptores para estes hormônios. Da mesma forma, a literatura mostra que o EE, um estrogênio sintético, com potência mil vezes superior à do estrogênio endógeno, induz alterações na cascata de coagulação, culminando no aumento dos fatores pró-coagulantes (fibrinogênio, VII, VIII, IX, X, XII e XIII) e na redução dos fatores inibitórios (proteína $S$ e antitrombina $)^{10}$. O progestagênio, ao contrário, quando ministrado isoladamente, não promove tais alterações ou afeta de forma mínima o sistema de coagulação.

Sabe-se ainda que o estrogênio dos ACH aumenta o risco de trombose arterial (IAM e AVE), especialmente em pacientes com fatores de risco cardiovascular - tabagismo, obesidade, hipercolesterolemia, HAS, DM. Isto se torna importante, pois, além da alteração dos hábitos de vida da atualidade - redução da ingesta de fibras, dieta rica em gordura e sedentarismo -, tem-se visto maior incidência de tais fenômenos, que antes eram raros durante a idade reprodutiva ${ }^{10}$.

O uso de estrogênio de baixa dosagem (<50 mcg) está associado a um risco cerca de duas vezes maior de trombose arterial, mesmo após a correção dos fatores de risco cardiovasculares, quando comparado ao risco de pacientes que usam qualquer método contraceptivo hormonal. Além disso, a literatura mostra que o risco de IAM é cerca de dez vezes maior em pacientes menores de 35 anos e tabagistas quando comparadas a não tabagistas. A associação de idade maior que 35 anos, tabagismo e uso de ACH combinado aumenta significativamente o risco de eventos trombóticos arteriais, o que torna, portanto, extremamente perigosa a prescrição desses fármacos. Já os progestágenos e a via de administração não influenciam significativamente esse aspecto ${ }^{10}$.

Da mesma forma, foi constatado que o risco de AVE aumenta com o uso de ACO combinado. Tal efeito deve-se, assim como no IAM, às altas dosagens de EE ( $\geq 50 \mathrm{mcg}$ ) das formulações, sendo a progesterona isenta de efeitos significativos. Ademais, migrânea com aura duplica o risco de AVE, quando comparado aos casos sem aura. Por esse motivo, nos casos de pacientes com diagnóstico de migrânea com aura, deve-se evitar a prescrição de contraceptivos hormonais combinados, podendo-se fazê-lo apenas com uso de compostos isolados de progestágeno, desde que a mulher não apresente outros fatores de risco para $\mathrm{AVE}^{10}$.

Outro ponto importante é a influência dos ACH na pressão arterial (PA). Estudos mostram que o EE, por ser bem mais potente que o estrogênio endógeno, aumenta a produção de angiotensinogênio hepático, o que culmina com o aumento (através do sistema renina-angiotensina-aldosterona) da PA em 2-3 mmHg em mulheres saudáveis. Os progestágenos, por sua vez, não influenciam significativamente esse aspecto. Por isso, para pacientes hipertensas, a OMS recomenda a prescrição de contracepção hormonal isolada com progestágeno ou métodos não hormonais, independentemente do grau da hipertensão. Os ACH combinados, além de aumentarem a PA, elevam o risco de trombose arterial em pacientes predispostas ${ }^{10}$.

A deficiência no ensino sobre o tema leva à formação de um conhecimento fragmentado sobre este assunto ${ }^{11}$. Considerando que o profissional médico é uma extensão dos acadêmicos de Medicina, em especial aqueles do internato, observa-se também que o efeito potencial dessa inconsistência de conhecimento é amplo, inclusive entre esses profissionais ${ }^{4}$. O resultado final dessa cascata é sentido pela paciente, para quem os métodos contraceptivos são, muitas vezes, pouco esclarecidos ou até mesmo indicados de forma incorreta ${ }^{10,11,12}$

Um estudo semelhante realizado na Universidade Federal do Piauí (UFPI) com 283 estudantes de Medicina e Enfermagem mostrou que apenas metade dos entrevistados tinha conhecimento adequado sobre os anticoncepcionais orais, o que vem ao encontro do que foi evidenciado no presente estudo ${ }^{11}$.

A literatura mostra ainda que a falta de conhecimentos a respeito da contracepção hormonal é maior nas escolas dos países em desenvolvimento, como o Brasil ${ }^{4}$. Esta situação também foi demonstrada neste estudo.

Não prescrever um ACH com estrógenos para as situações especiais investigadas neste estudo é importante para a segu- 
rança da mulher. Contudo, deixar de prescrever um método hormonal de progestágeno isolado, que não é contraindicado nessas situações, talvez seja ainda mais grave porque deixa essas mulheres em situação de vulnerabilidade para uma gestação indesejada ou não planejada e de muito mais alto risco para a saúde materno-infantil. Na gravidez, sabidamente, as dosagens dos esteroides sexuais são muito maiores do que no $\mathrm{ACH}$, o que se associa a maior mortalidade materna e perinatal.

Sabe-se ainda que os efeitos hormonais da gestação são de maior morbidade do que os efeitos dos contraceptivos ministrados, devido a essa diferença de quantidade hormonal a que a mulher está exposta durante a gestação. Nesse sentido, conclui-se que, em situações mais graves - tromboembolismo pulmonar e eventos trombóticos e arteriais prévios, cefaleia com aura e tabagismo em pacientes com mais de 35 anos -, o risco de eventos trombóticos arteriais e venosos é maior durante a gestação do que durante o uso desses métodos hormonais ${ }^{12}$.

Conclui-se, portanto, que os conhecimentos dos internos de Medicina, aqui estudados, sobre contracepção hormonal em situações especiais, de acordo com os critérios de elegibilidade da OMS, são fragmentados e estão bem aquém do esperado para que eles possam orientar e prescrever com segurança anticoncepcionais hormonais em situações que envolvam anticoncepção hormonal com hipertensão, migrânea, tabagismo e tromboembolismo.

Os achados do presente estudo sinalizam a necessidade de uma revisão do plano de ensino médico na escola estudada (PUC-GO), para que se dedique mais atenção a esse tema. Somente assim será alcançado o patamar da efetiva promoção de saúde e qualidade de vida para a população, em especial a materno-infantil.

\section{REFERÊNCIAS}

1. Burkman RT, Collins JA,Shulman LP,Williams JK. Current perspectives on oral contraceptive use. Am J Obstet Gynecol. 2011;185(2).

2. Drey EA, Darney PD. Recent developments in hormonal contraception. Rev Endocr Metab Disord 2002; 3(3):257265.

3. Swyer GI. Advances in hormonal contraception. Practitioner 1973; 211(264):535-540.

4. Schreiber CA, Harwood BJ, Switzer GE, Creinin MD, Reeves MF, Ness RB. Training and attitudes about contraceptive management across primary care specialties: a survey of graduating residentes Contraception 2006; 73:618-622.

5. Spitzer WO. Bias versus causality: Interpreting recent evidence of oral contraceptive studies. Am J Obstet Gynecol. $1998 ; 179(3)$.
6. Goldzieher JW, Thirty years of hormonal contraception: a historical perspective. Int J Fertil,1991, 36 (Suppl 3):10-15.

7. Rowen TS, Smith JS, Eisenberg ML, Breyer BN, Drey EA, Shindel AW. Contraceptive usage patterns in North American medical students. Contraception 83, p 459-465, 2011.

8. Weir RJ, Briggs E, Mack A, Naismith L, Taylor L, Wilson E.Blood pressure in women taking oral contraceptives. $\mathrm{Br}$ Med J 1 (5907):533-535, 1974.

9. World Health Organization. Medical elegibility criteria for contraceptive use. 4.ed,. Genebra, :WHO; 2009.

10. Brito MB, Nobre F,Vieira CS. Contracepção hormonal e sistema cardiovascular. Sociedade Brasileira de Cardiologia, MCMXLIII; 2010.

11. Seabra LO, Nery IS, Moreira FHB, Rocha JS, Gonçalves LRR. Conhecimento de métodos contraceptivos por universitários da área da saúde. UFPI. Teresina, 2012

12. Batarda MARS, Macedo AF. Tromboembolismo Venoso associado à Contracepção Hormonal Combinada. Prescrição adequada ao risco individual? Universidade da Beira Interior, Covilhã: 2010.

\section{CONTRIBUIÇÃO DOS AUTORES}

Margareth Rocha Peixoto Giglio: contribuição científica e intelectual efetiva para o estudo; concepção e delineamento; interpretação dos dados; procedimentos técnicos; análise estatística; preparação do manuscrito; redação do manuscrito; revisão crítica; aprovação final. Larissa Cruvinel Andrade: contribuição científica e intelectual efetiva para o estudo; concepção e delineamento; aquisição dos dados; interpretação dos dados; procedimentos técnicos; análise estatística; preparação do manuscrito; redação do manuscrito; aprovação final. Giovana Mahamed Daher: contribuição científica e intelectual efetiva para o estudo; concepção e delineamento; aquisição dos dados; interpretação dos dados; procedimentos técnicos; análise estatística; preparação do manuscrito; redação do manuscrito; aprovação final. Marilia Oliveira Ribeiro: revisão crítica. Marco Aurélio Albernaz: revisão crítica.

\section{CONFLITO DE INTERESSES}

Os autores declaram não haver conflitos de interesse.

\section{ENDEREÇO PARA CORRESPONDÊNCIA}

Departamento de Medicina da PUC

Margareth Rocha Peixoto Giglio

Avenida Universitária, 1440 - Setor Universitário - Goiânia CEP 74605-010 - GO

E-mail: margarethgiglio@uol.com.br 